Review Article

\title{
Epidemiology of Alzheimer's Disease and Dementia in Arab Countries: A Systematic Review
}

\author{
Ashraf El-Metwally (D, ${ }^{1}$ Paivi Toivola, ${ }^{2}$ Mashael Al-Rashidi, ${ }^{3}$ Shanila Nooruddin, ${ }^{4}$ \\ Munazza Jawed, ${ }^{4}$ Raghad AlKanhal, ${ }^{5}$ Hira Abdul Razzak, ${ }^{6}$ and Nada Albawardi ${ }^{7}$ \\ ${ }^{1}$ College of Public Health and Health Informatics, King Saud Bin Abdulaziz University for Health Sciences, Riyadh, Saudi Arabia \\ ${ }^{2}$ King Abdullah Specialist Children Hospital, King Abdulaziz Medical City, Riyadh, Saudi Arabia \\ ${ }^{3}$ Saudi Food and Drug Authority, Riyadh, Saudi Arabia \\ ${ }^{4}$ Dow University of Health Sciences, Karachi, Pakistan \\ ${ }^{5}$ King Abdullah International Medical Research Center/King Saud bin Abdulaziz University for Health Sciences, Ministry of National \\ Guard-Health Affairs, Riyadh, Saudi Arabia \\ ${ }^{6}$ Ministry of Health and Prevention, Dubai, UAE \\ ${ }^{7}$ Epidemiology and Biostatistics Section, Health Sciences Research Centre, Princess Nourah bint Abdulrahman University, \\ Riyadh, Saudi Arabia
}

Correspondence should be addressed to Ashraf El-Metwally; elmetwally.ashraf@outlook.com

Received 26 January 2019; Revised 25 May 2019; Accepted 16 September 2019; Published 29 October 2019

Academic Editor: Luigi Trojano

Copyright (c) 2019 Ashraf El-Metwally et al. This is an open access article distributed under the Creative Commons Attribution License, which permits unrestricted use, distribution, and reproduction in any medium, provided the original work is properly cited.

Background/Objectives. Contrary to popular belief, the condition of dementia is not an actual discrete disease, but rather a group of symptoms, most notable of which is the disturbance of memory and social ability, often severe enough to impair daily functioning. As a result, it has been a major cause of functional deterioration among varying populations in the world. This study is aimed at reviewing the epidemiology of dementia in Arab countries in terms of its prevalence, distribution, and risk factors. Methods. A systematic literature review was conducted using articles published in PubMed, Embase, Scopus, and other local journals between 1990 and 2018. After applying the inclusion and exclusion criteria, a total of 18 studies were concluded to be eligible for the review. Results. Prevalence studies demonstrated that dementia is indeed a prevalent condition in Arab countries, ranging between $1.1 \%$ and $2.3 \%$ among age groups of 50 years and older, as well as between $13.5 \%$ and $18.5 \%$ among age groups of 80 years and above. However, these results are not different from those of many other countries in the world. Moreover, prevalence was found to vary depending on sociodemographic characteristics. Major risk factors of dementia included hypertension, low income, and low education, while the risk of developing dementia is increased by obesity, diabetes mellitus, and cardiovascular risk factors. Despite the growing evidence regarding the epidemiological distribution and determinants of dementia worldwide, studies from the Arab region remain scarce. Conclusion. This systematic review highlights the need for population-based studies to provide necessary information for developing preventive and curative strategies specific to the Arab region.

\section{Introduction}

Alzheimer's disease (AD) and its related disorders are conditions characterized by a gradual and unpreventable deterioration of multiple cognitive functions (including complex attention, executive function, learning and memory, language, perceptual-motor, or social cognition from a previous level of performance), serious enough to interfere with the daily lives of those affected in both social and professional terms $[1,2]$. One of the special characteristics of $\mathrm{AD}$ is dementia, a "neurocognitive disorder" or NCD, according to DSM-5. These disorders indubitably have a major negative impact on the lives of the patients, their families, and society $[3,4]$. The World Health Organization (WHO) determined 
that dementia can currently be found among 36 million of the world's population with a progressive increase in occurrence over the past decades. The WHO also estimates that this number will be doubled within the next 20 years [5]. Similarly, the global number of AD patients was 44 million in 2015; however, this number is expected to triple, reaching 115 million individuals by 2050 [6]. Every year, around 4.6 million new dementia cases are added in the existing pool, the highest growth projections being found in China along with its South Asian neighbors [7]. Nevertheless, previous evidence indicates that dietary and lifestyle modification could reduce the risk of dementia and its comorbidities such as type 2 diabetes, hypertension, obesity, and psychiatric behavioral changes $[8,9]$.

Epidemiological research studies have constantly shown differences in the rates of dementia among different geographical and ethnic populations and other sociodemographic characteristics. Compared to countries in Europe and North America, lower dementia rates are reported in countries such as those in Africa and Asia, specifically Singapore, Japan, China, Nigeria, and India [10]. In the United States, the occurrence of $\mathrm{AD}$ was found to be $1.6 \%$ among the $65-74$-year age group, followed by $19 \%$ in the $75-84-$ year age groups and up to $42 \%$ among those who are 85 years old and above [11]. Thus, it can be easily concluded that one of the most significant risk factors for dementia is age [1]. The WHO also reported that the average aging population's ( 85 years and more) growth rate will increase by $5 \%$ between 2000 and 2050 in some Arab countries [12]. Therefore, age differences between populations often result in varying estimates of the prevalence, making it difficult to accurately compare them. Furthermore, vascular risk factors and genetic factors (apolipoprotein E) also play a significant role. Indeed, the prevalence of dementia among the elderly is significantly higher than that among individuals aged 20 to 50 [1]. A meta-analysis showed strong evidence for the association of dementia with statin, estrogen, nonsteroidal anti-inflammatory drugs (NSAIDS), and antihypertensive medications, while folate, coffee, and vitamins $\mathrm{C}$ and $\mathrm{E}$ were found to be protective for $\mathrm{AD}$ [13]. Moreover, depression, hypertension, atherosclerosis, arthritis, and diabetes significantly increase the risk of AD. Likewise, lifestyle factors including high BMI and low levels of education were among other risk factors identified. On the other hand, current smoking, cognitive activity, and light-to-moderate drinking decrease this risk [13].

Epidemiological studies have made it possible to highlight the complexity of these disorders and to identify major characteristics that have a direct influence on their distribution. Like other countries in the world, the Middle East has undergone many considerable changes that have had a direct influence on the population's health issues. One major change highlighted by Simpson [2] is that noncommunicable diseases replaced infectious diseases as the main health concern within the region. Findings reveal that $47 \%$ of the total burden of disease is attributed to noncommunicable diseases, with a steep rise to $60 \%$ expected by 2020 [3]. A limited amount of data is currently available from Middle Eastern countries about Alzheimer's and dementia. Moreover, no clear evidence for the occurrence of $\mathrm{AD}$ following the increase in the aging population has been generated.

A dementia report from the WHO has revealed an alarming increase in dementia across the Middle Eastern region, where prevalence may have an increase of $125 \%$ by 2050 [14]. Several studies have been conducted in Arab countries to obtain data on the prevalence, risk factors, prevention, and management of $\mathrm{AD}$ and dementia, but no review had yet been conducted to systematically summarize current existing evidence. Therefore, we aimed to fill this gap in the literature by carrying out a systematic review to evaluate the epidemiology of dementia in Arab countries, with a clear focus on its prevalence, distribution, and risk factors.

\section{Methods}

2.1. Data Collection Strategy. Studies from 1990 to 2018 were systematically retrieved and extracted from PubMed, Scopus, and Embase. Detailed searches in local journals and cross-referencing were also performed to enrich the investigation and to broaden the scope of the research. A combination of Medical Subject Heading terms and search terms were utilized, including "AND" and "OR" Boolean operators to retrieve the targeted results. The key terms used in the search include "Epidemiology"; "Prevalence"; "Complications"; "Risks"; "Dementia"; "Alzheimer's Disease (AD)"; "Arab Countries"; "Arab World"; "Incidence"; "Prognosis"; "Saudi"; "Kuwait"; "Egypt"; "Syria"; "Lebanon"; "Libya"; "Sudan"; "Algeria"; "Jordon"; "Tunisia"; "Iraq"; "Morocco"; "Qatar"; and "Bahrain."

2.2. Study Eligibility Criteria. An inclusion criterion was applied to the article title and abstract. Articles providing information on the prevalence or risk factors of dementia and Alzheimer's disease in one or more selected Arab regions were included. Additionally, primary research articles published in English in peer-reviewed journals between the late 1990s and 2018 were extracted to be included in the study. On the other hand, studies including nonrelevant regions and populations were excluded.

2.3. Identification and Study Selection. Research studies were selected and reviewed using a three-step process. Firstly, two of the researchers independently selected the studies that matched the standards for selection by browsing through the retrieved abstracts and titles. Secondly, the two researchers proceeded to discretely read potentially eligible studies. The outcome of these steps of the review demonstrated no disagreements or inconsistencies within the selection of studies. Finally, the lists of references of the studies included were scanned to identify additionally relevant research papers, completing the third step of the process. The selection of articles followed the Preferred Reporting Items for Systematic Reviews and Meta-Analyses (PRISMA) guidelines.

2.4. Data Analysis. A total of 352 articles (116 from PubMed, 134 from Embase, 99 from Scopus, and 3 through crossreferencing/local journals) were identified in the original search. Articles that formed duplicates were then removed, 
and the remaining papers were further scanned based on their relevance to the topic. To apply the inclusion criteria, titles and abstracts of each article were reviewed thoroughly. All articles with insufficient information, unrelated topics, or nonrelevant populations were excluded. A total of 53 full-text articles were assessed for eligibility. Finally, we were able to select 18 pertinent articles that conformed to the inclusion criteria and provided succinct information which met the aims and objectives of the study. Afterwards, the data collected was summarized through narrative with an overview of the year, country, outcome measure, setting/sampling technique, study design, sample size, and study key findings. This was then followed by a synthesis of the selected studies based on the outcome measures. Figure 1 represents the process of the research.

2.5. Risk of Bias Assessment. We adopted the quality assessment score published by Prince et al. [15]. It includes four quality assessment norms with a focus on the key areas leading to potential bias in observational studies, including sample size, study design, response proportion, and diagnostic assessment. The overall quality was assessed by summing the scores up. Sample size estimation is undoubtedly an important factor for the study design; it should be sufficient for a proper estimation of the prevalence for a geographic area in published studies. Similarly, the study population should also be correctly classified based on the conditions present with a specified confidence interval and sampling error [16].

\section{Results and Discussion}

3.1. Overview of the Included Studies. The PRISMA flowchart (Figure 1) presents the results of the screening and selection process of the articles identified. The systematic literature search retrieved 352 studies, of which 18 were eventually found to meet the objectives of the review. These studies were sorted into different categories depending on prevalence, frequency of sub-types, and associated risk factors. Thirteen of the studies reported on the prevalence and frequency of dementia in the Middle East, four of which were hospital-based studies. Furthermore, eleven of the publications discussed the risk factors associated with dementia (Tables 1, 2, and 3).

3.2. Quality Assessment of the Included Studies. Out of the eighteen studies which were included in this systematic review, only five studies had a sample size of more than 1,500 , while almost half of the studies had a sample size of less than 500. Most of the studies were conducted in multiple stages, but they had no samplings of screen negatives or weighing back. Only $50 \%$ of the studies applied proper sampling techniques. Studies by El Tallawy et al. [18] involved door-to-door screenings of individuals with a maximum sample size of the general population. About $30 \%$ of the studies did not mention the response rate, while others extracted data from the hospital records retrospectively. Regarding the diagnostic assessment, around $45 \%$ of the studies involved multiple strategies for better and more definite diagnoses of dementia and Alzheimer's disease. For the reviewed studies, we gave the quality score ranging from 0 to 11 . The studies were categorized as low quality (score $0-4$ ), medium quality [5-8], and high quality with scores [9-11]. Overall, the studies from Egypt, Saudi Arabia, and UAE showed the highest scores (Table 4).

3.3. Prevalence. Studies about the prevalence and frequency of dementia and its subtypes were conducted across six countries; four studies in Egypt [17-20], two in Lebanon [21, 22], three in Saudi Arabia [3,26,27], two in Qatar [23, 28], one in Tunis [24], and one in the United Arab Emirates (UAE) [25]. These studies were published between 1990 and 2018 and included sample sizes ranging from 77 to 62,583 subjects. The settings for the studies included households, nursing homes, and healthcare facilities. The methodologies used were heterogeneous from the use of cross-sectional studies with a single phase of screening or diagnostic testing using only interviews or questionnaires to multistage testing comprising clinical examination, laboratory, and radiological investigations. Other studies reviewed hospital records of both in-patient and out-patient departments and described hospital frequencies of dementia and its subtypes. Study populations also varied widely among different research articles, with some including all members of households and others limiting evaluations to those over 50 or 60 years of age.

\subsection{Risk Factors}

3.4.1. Age. The association between age and dementia was reported in five studies. A secondary analysis was conducted using random sample data of elders from two Lebanese governorates in a cross-sectional study [22]. The study reported significantly increased odds of dementia with increased age [OR: $75-84$ years $=4.00$ (95\% CI: $1.46,10.95)$; OR $85+$ years $=7.07(1.84,27.03)]$ [29]. Similarly, two other crosssectional studies revealed age-specific dementia prevalence, which was projected to double every 5 years in a sample from Egypt [17]. On the other hand, Ouanes et al. [24] further noted no significant association between age and the prevalence of dementia among nursing home residents in Tunisia.

3.4.2. Education. Education was reported to be significantly associated with dementia in four studies. El Tallawy et al. [19], who conducted a cross-sectional study among $\geq 50$ year-old Egyptians, reported a significantly higher prevalence of dementia among illiterate participants compared to educated ones (3.6\% vs. $79 \%$ respectively, $p<0.001)$. In another cross-sectional study, Khedr et al. [20] reported a greater prevalence of dementia in illiterate elderly participants as opposed to their literate counterparts $(10.12 \%$ vs. $2.25 \%$; $p=0.00001$ ) within a random sample of households in Egypt.

In one particular case, which was a control study using subjects from senior homes and a university hospital in Jordan, Al-Khateeb et al. [30] found a 6.29-fold ( $p=0.002)$ rise in the risk of developing dementia for illiterate subjects. In another cross-sectional study, Chaaya et al. [29] reported that the odds of dementia were significantly higher (OR: 3.39, 95\% CI: $1.71,6.70)$ among those with no formal education in Lebanon (formal education including those who went to 

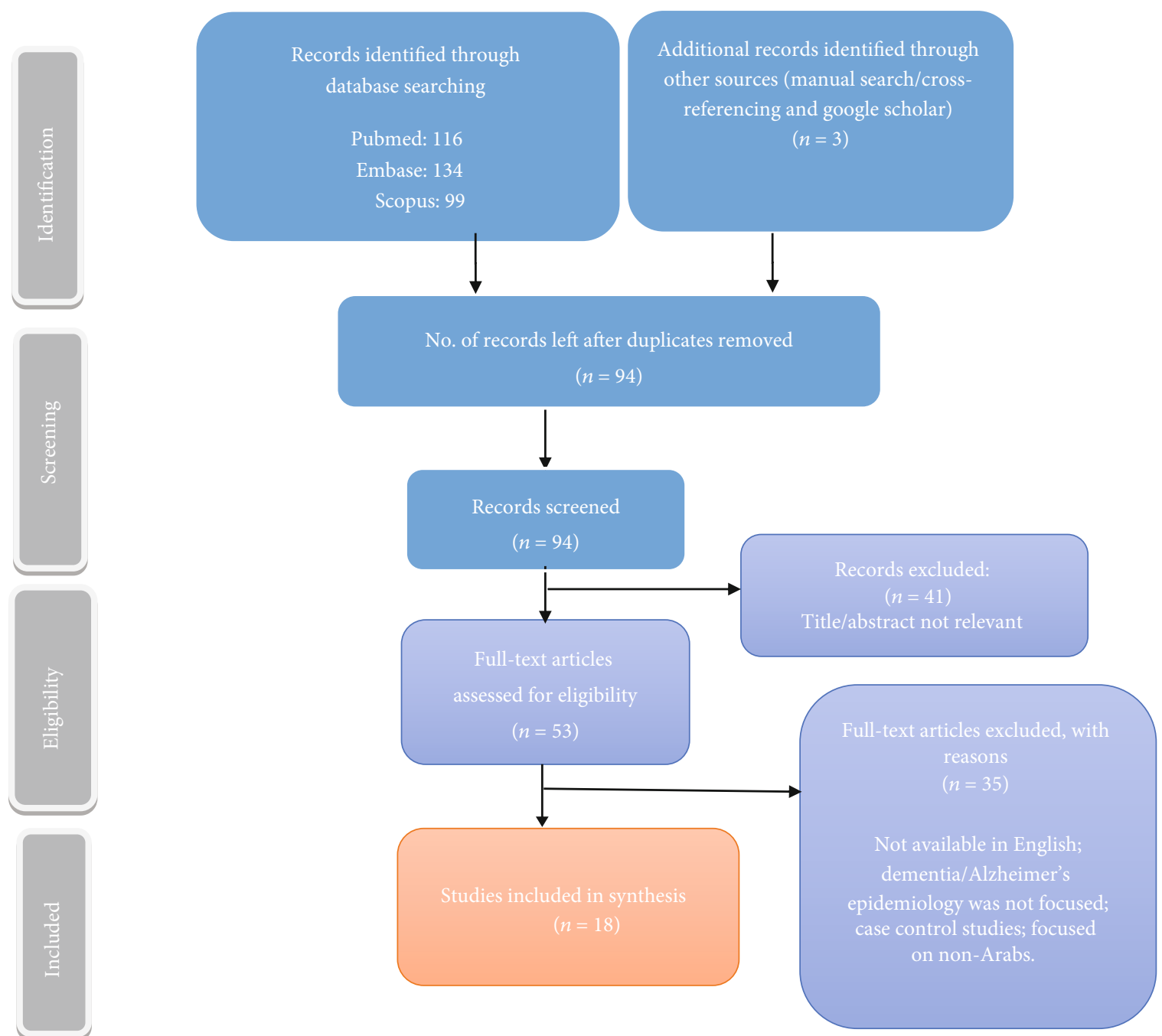

FIGURE 1: Retrieval of article and screening process.

school, technical institute, or university vs. no formal education or no enrollment in any educational system including those who read and write or were illiterate). Only one cross-sectional study reported that education level was not significantly associated with dementia [24].

3.4.3. Gender. Two cross-sectional studies reported on the association of dementia and gender. Chaaya et al. [29] found that being female increased the odds of developing dementia by no less than 2 times. However, when controlling for all other factors (such as, age, marital status, education, and income), the outcomes were not statistically significant. Likewise, Ouanes et al. [24] found that gender did not differ significantly between nursing home residents with or without dementia.

3.4.4. Health Conditions. Chaaya et al. [29] assessed the association between dementia and cardiovascular disease (CVD) risk factors and found that uncontrolled hypertension (vs. no hypertension) was the sole significant risk factor that exacerbated the odds of developing dementia [OR 6.35 (95\% CI:
1.60, 25.10)] among elderly participants. Among elderly Saudi subjects from an outpatient clinic, Alaama et al. [31] reported in a cross-sectional study that diabetics were significantly more likely to have cognitive impairment than those who were not diabetic $(16 \%$ vs. $3 \%, p<0.005)$. Moreover, no statistically significant difference was found among diabetic patients for glycemic control or the duration of diabetes on cognitive test results. In a case-control study, Al-Khateeb et al. [30] also demonstrated no significant correlation between serum copper, lipid profile, and cognitive decline among elderly Jordanians.

3.4.5. Genetic Factors. Haithem et al. [32] conducted a case control study in Tunisia to examine the association between five genetic risk factors for dementia, studied separately or assembled in haplotypes. All the polymorphisms studied, excluding PON1-Q192R, were found to be significant. Shamieh et al. [34] explored the distribution of APOE genotypes within the general Lebanese population in a cross-sectional study. They found that the prevalence of these genotypes in Lebanon was similar to that in Asian 


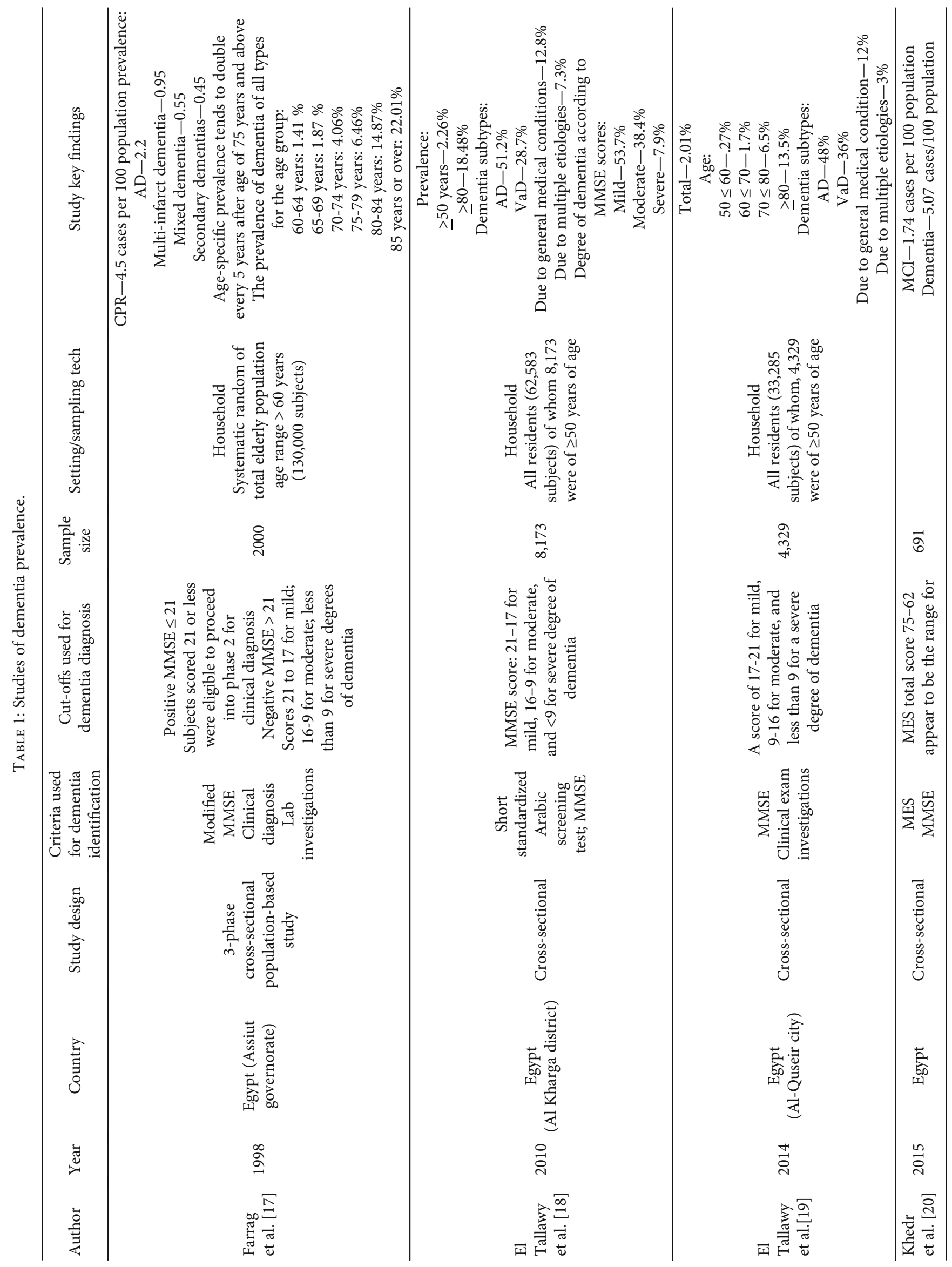




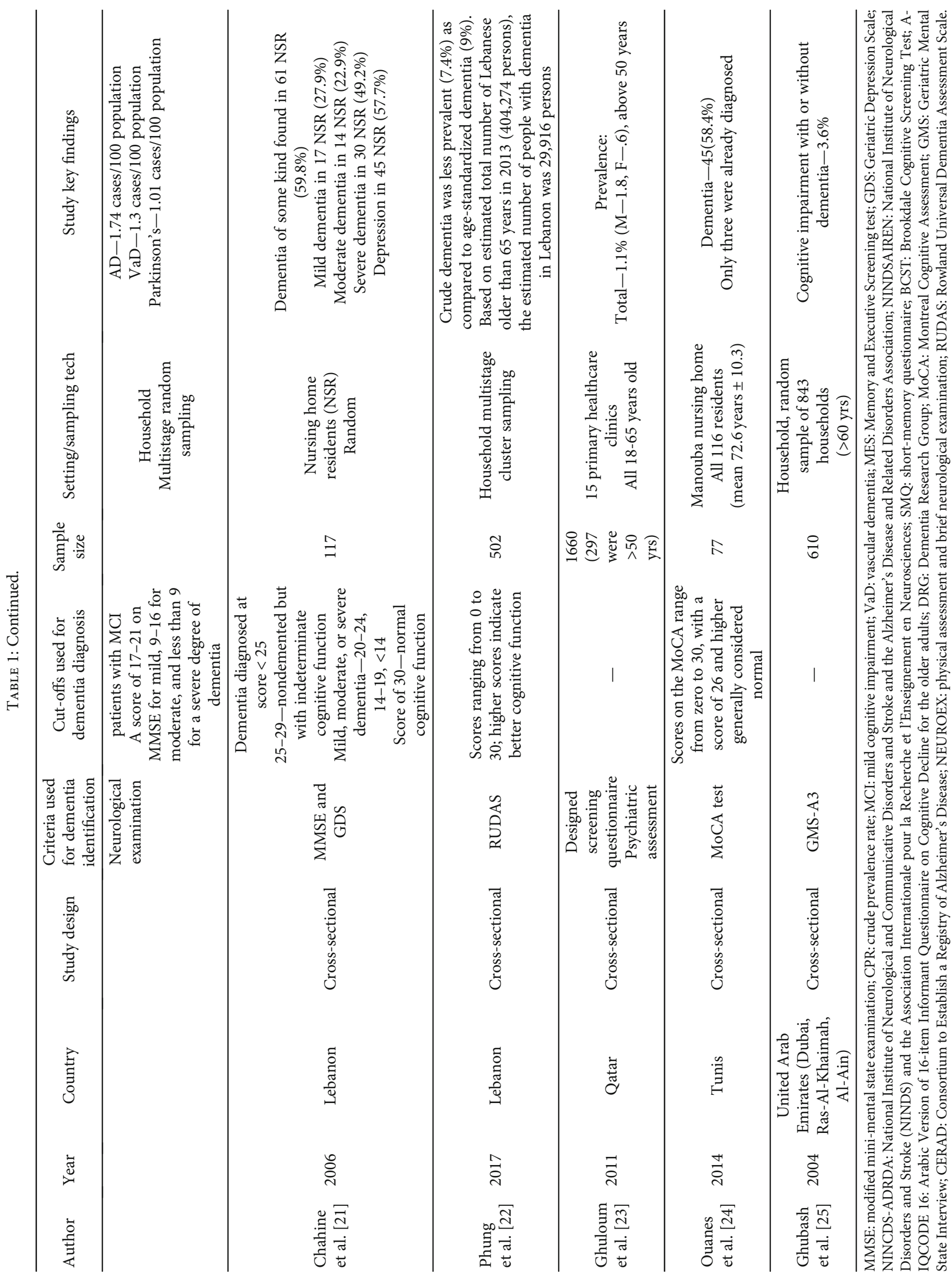




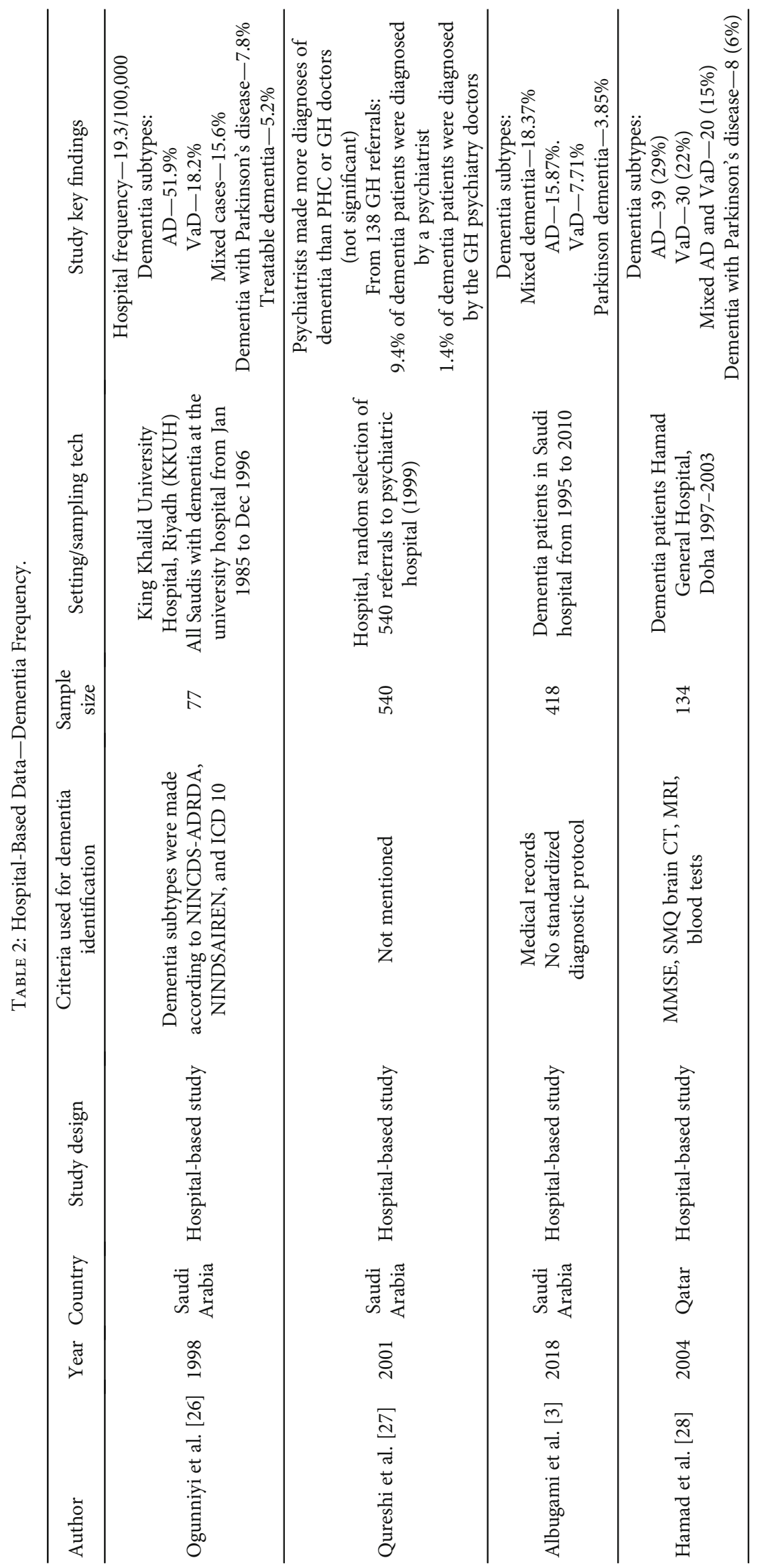




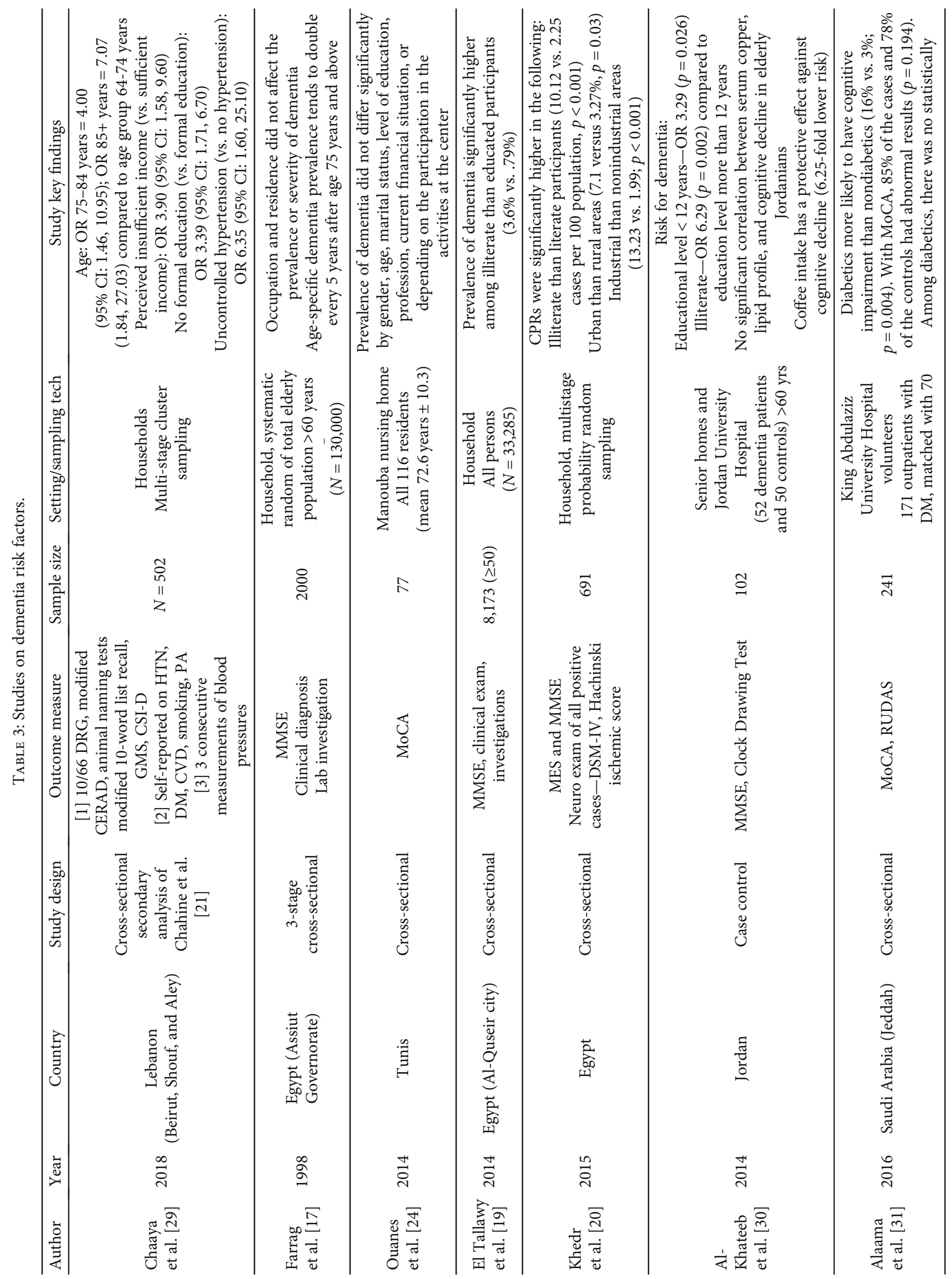




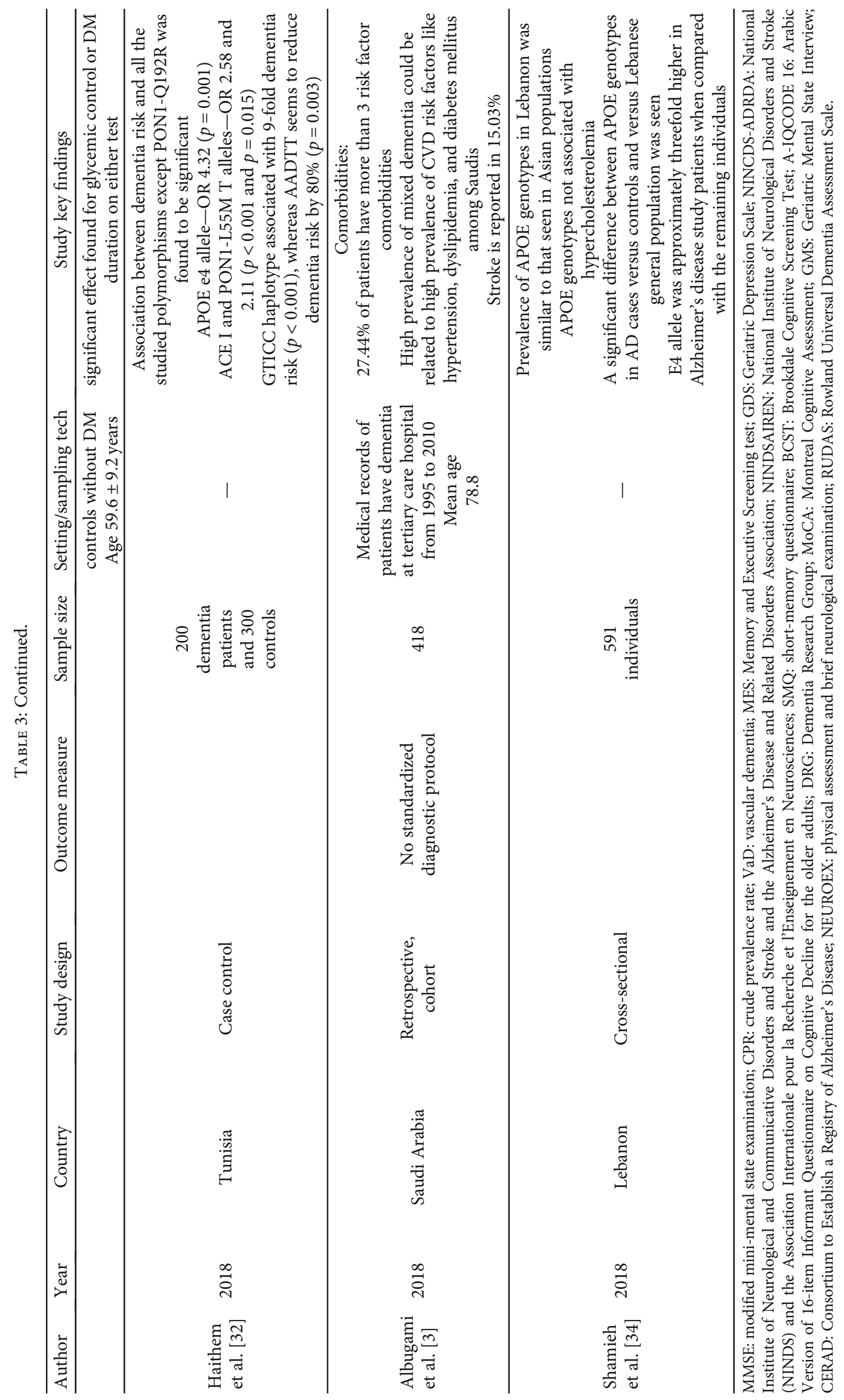


TABLE 4: Quality assessment of the included studies.

\begin{tabular}{|c|c|c|c|c|c|}
\hline Author & Sample size & Study design & Response proportion & Diagnostic assessment & Total \\
\hline Farrag et al. [17] & 1.5 & 2 & 3 & 2 & 8.5 \\
\hline El Tallawy et al. [18] & 2 & 2 & 3 & 4 & 11 \\
\hline El Tallawy et al. [19] & 2 & 2 & 3 & 4 & 11 \\
\hline Khedr et al. [20] & 1 & 2 & 3 & 4 & 10 \\
\hline Chahine et al. [21] & 0.5 & 2 & 1 & 1 & 4.5 \\
\hline Phung et al. [22] & 1 & 0 & 1 & 4 & 6 \\
\hline Ogunniyi et al. [26] & 0.5 & 0 & 0 & 2 & 2.5 \\
\hline Qureshi et al. [27] & 1 & N/A & N/A & 3 & 4 \\
\hline Albugami et al. [3] & 0.5 & 2 & 3 & 4 & 9.5 \\
\hline Hamad et al. [28] & 0.5 & 2 & 0 & 4 & 6.5 \\
\hline Ghuloum et al. [23] & 1.5 & 1 & 2 & 2 & 6.5 \\
\hline Ouanes et al. [24] & 0.5 & 0 & 2 & 1 & 3.5 \\
\hline Ghubash et al. [25] & 1 & 2 & 3 & 4 & 10 \\
\hline Chaaya et al. [29] & 1 & 0 & 1 & 4 & 6 \\
\hline Al-Khateeb et al. [30] & 0.5 & 0 & 0 & 1 & 1.5 \\
\hline Alaama et al. [31] & 0.5 & 0 & 0 & 1 & 1.5 \\
\hline Haithem et al. [32] & 0.5 & 0 & 0 & 1 & 1.5 \\
\hline Shamieh et al. [34] & 1 & 0 & 0 & 1 & 2 \\
\hline
\end{tabular}

Sample size: $<500,0.5$ point; 500-1499, 1 point; 1500-2999, 1.5 points; $\geq 3000,2$ points. Study design: two-phase or one-phase study with no sampling of screen negatives, 0 points; two-phase study with sampling of screen negatives but no weighting back, 1 point; one-phase study or two-phase study with appropriate sampling and weighting, 2 points. Response proportion: not mentioned, $0 ;<60 \%, 1$ point; $60-79 \%, 2$ points; $\geq 80 \%$, 3 points. Diagnostic assessment: one point each for multidomain cognitive test battery, formal disability assessment, informant interview, and clinical interview.

populations. There was a significant difference in APOE genotypes in $\mathrm{AD}$ patients, controls, and the general Lebanese population, where the $\mathrm{E} 4$ allele was 3 times higher in $\mathrm{AD}$ patients.

3.4.6. Other Factors. Other variables associated with cognitive decline or risk for dementia included insufficient personal income (OR: 3.90, 95\% CI: 1.58, 9.60) [29], while in another cross-sectional study, occupation and residence had no significant effect on the prevalence of dementia [17]. Regular consumption of coffee (drinking coffee nearly every day) showed a protective effect of a 6.25-fold decrease in the risk of cognitive decline [30]. In Egypt, Khedr et al. [20] reported that the CPRs were considered to be more significant in urban than in rural areas (7.1 versus 3.27/100, respectively; $p=0.03)$ and in industrial areas than in nonindustrial areas (13.23 versus $1.99 ; p \leq 0.001$ ).

3.5. Discussion. In the current systematic review, we aimed to evaluate the prevalence, distribution, and risk factors of dementia and $\mathrm{AD}$ in Arab countries. A total of 18 studies were included in this review. Among them, five were classified as high quality, six as medium quality, and seven as low-quality studies according to our assessment criteria.

Dementia and its subtypes were found to be prevalent in Arab countries as shown by our systematic review. However, there is a lack in population-based studies in the Arab world. There was a lower number of studies that examined the prevalence and risk factor of dementia and AD in most of the Arab countries, with particularly limited estimates for recent years. One of the most common risk factors associated with it was age. High occurrence of cognitive decline, AD, and dementia were found to be significantly associated with increasing age. Besides age, another notable risk factor was education, showing higher odds of dementia in illiterate compared to educated people. Moreover, females were at a higher risk of developing dementia, $\mathrm{AD}$, and cognitive decline than males. Health conditions of the patients like hypertension, cardiovascular diseases, diabetes, and depression were also among the identified risk factors for dementia in Arab countries. This review also picked a few other factors which were found to lower the odds of developing dementia, namely, regular utilization of coffee and smoking.

The reviewed literature on the prevalence of dementia and $\mathrm{AD}$ in various Arab countries shows that Egyptian studies reported a prevalence of dementia that was around 2$2.26 \%$ [17-20], 3.34-7.4\% in Lebanon [21, 22], 5.2-3.85\% in the KSA [3, 26, 27], $1.1 \%$ in Qatar [28], 58.4\% in Tunisia [24], and 3.6\% in the UAE [25]. The literature includes various other studies that demonstrated the prevalence of $\mathrm{AD}$ and dementia in their respective populations. The estimates of prevalence of vascular dementia in Latin America are $0.9 \%$ for a study in Brazil [35], 2.1\% in Venezuela [36], and $1.9 \%$ in Cuba [37], while similar frequencies were observed between $0.6 \%$ in Sri Lanka and 2.1\% [38] in South Korea [39]. In a study from Istanbul, AD prevalence was $11 \%$, specifically among people aged 70 years and above [40], whereas more recent evidence shows a $6.4 \% \mathrm{AD}$ prevalence, as reported in a study conducted on the Turkish population [41]. Similarly, a study conducted on the Arab population 
of Israel showed a higher prevalence rate of vascular dementia 5.9\% [42]. Ertekin et al. [41] further point out that AD is more common in elderly. This is fully justified because individuals belonging to the 75-84-year age group had ten times the $\mathrm{AD}$ prevalence of those aged 65-74 years. Moreover, the risk of developing $\mathrm{AD}$ for people aged 85 years and above was 36 times higher than that of the 65-74 age group, with an overall $\mathrm{AD}$ risk to be increased 17 -fold with advancing age. The major driver of this projected increase is population aging. On a similar note, a meta-analysis including 17 studies concluded that the occurrence of dementia was $2.2-8.4 \%$ at 65 years old and over, $10.5-16 \%$ at 75 years old and above, and $15.2-38.9 \%$ at 85 years old and over [37]. Other drivers for this projected increase may include lack of knowledge about dementia, low literacy levels, and culture. Elderly adults are highly respected within Arab culture, and placing one's elderly relatives somewhere outside their home is considered an abandonment of family duty. Often, the dementia carries stigma with it. Therefore, it is widely believed that dementia is caused by fate.

$\mathrm{AD}$ is more common in women than in men [41]. Incidence studies for dementia report no difference of risks between males and females [43]. However, the prevalence studies have shown females to be more vulnerable to developing dementia $[1,44]$. Another large population-based study proposed no specific gender differences in the dementia incidence, yet after 90 years of age, the AD occurrence was higher in women, while the vascular dementia proportion was found to be higher in males than in females across all age groups [45]. Additionally, having a lower level of education was also recognized as one of the most established risks for dementia and $\mathrm{AD}$ [46]; this risk being similar in males and females [47].

Bowirrat et al. also report associated risk factors of dementia among the Arabs of Israel, which were being female, older age, and illiteracy [48]. Effects of education were assessed in very heterogeneous ways across studies (such as formal or no formal education; illiterate vs educated; $<12$ vs $>12$ years of education), and therefore, we could not compare their results. But as most researchers used years of education, we can say that dementia and years of education are inversely related to each other. Similar relation was also found in different population studies like Dong et al. [49], Ott Alewijin et al. [50], and Kalaria et al. [51]. These finding are consistent within Arabs living in non-Arab countries [52]. Drinking 3 to 5 cups of coffee per day was associated with a $65 \%$ reduced risk of $\mathrm{AD} /$ dementia at a later stage [53]. The Rotterdam study suggested that smokers have a greater risk of dementia and AD [54]. Depression was also proposed to be a risk factor and a prodrome of dementia [55].

This review also demonstrated the enhanced risk of developing $\mathrm{AD}$ in preexisting chronic diseases. Likewise, an increase in the rate of AD was noted by Ertekin et al. [41]. The authors revealed a 4.1-fold increase among diabetics, a 3.9-fold one among those with Parkinson's disease, and a 2.6-fold one in cardiac patients, as well as a 4.2 -fold increase in those people who had a history of dementia in their families. Saedi et al. [56] supplied strong evidence on the association of diabetes with increased risks of dementia and cognitive impairment. Biessels et al. [57] and Kravitz et al. [58] provided considerable insights on dementia as the most chronic complication of diabetes mellitus, occurring commonly at an older age [59]. The association between vascular dementia and diabetes was found to be $100-160 \%$ as compared to $\mathrm{AD}$ (45\%-90\%) [60], further advancing the danger of cerebrovascular accidents [61]. Ojo and Brooke [62] also evaluated the shared pathogenesis of $\mathrm{AD}$ and diabetes and argued about the compromised overall quality of life through weakening self-management that continues in a vicious cycle.

The study also reported a significant association of cardiovascular risk factors and the prevalence of dementia. The risk and incidence of dementia increase when a person is prone to smoking, alcohol, and obesity. These results are comparable with the studies conducted by Deckers et al. [63] and Suemoto [33]. Bowirrat et al. [48, 64] stated that higher prevalence rates of dementia in a certain population can be due to genetic factors, which undeniably form the major risk factor for the incidence of the disease in that given community. Similarly, another study on the Arab population living in Wadi Ara reported higher odds of Alzheimer's disease associated with hypertension $(\mathrm{OR}=2.08 ; 95 \% \mathrm{CI}$ : 1.18-3.65) [65]. Suemoto [33] argued that with aging, the risk of diagnosing vascular dementia among the elderly is reduced, but the risk of developing $\mathrm{AD}$ increases. Therefore, the vascular factor is especially dangerous across Arab countries, in which a significant part of the population simply does not survive the onset of dementia of a different type [66]. According to Rizzi et al. [67], mixed dementia results when cerebrovascular pathologies combine with AD. This implies that each pathology has its own effect on the continuum for dementia, whereby the pure $\mathrm{AD}$ and pure cerebrovascular disease form the two extremities of it.

3.5.1. Strengths and Limitations. Although adequate efforts have been made to include, retrieve, and search for all relevant articles, we were still unable to absolutely claim to have unearthed all potentially relevant articles for inclusion in this review. The exclusion of studies that were available in languages other than English also served as a limitation. Publication bias, which refers to the tendency to publish positive findings of the manuscripts, is a potential limitation of our study. The different factors responsible for causing variations in the results of selected studies such as genetic factors, methodological issues, socioeconomic characteristics, and the mortality rates among elderly population are also considered limitations. The more variation found between the studies in terms of population setting, etc., the more difficult it would be to compare these studies and combine their results for analysis. Traditionally, many studies explored the occurrence and epidemiology of dementia, but this review is the first of its kind to combine the findings systematically among different Arab countries.

\section{Conclusion}

This paper provided an overview of the burden of dementia in Arab countries. There have been only a limited number of primary studies conducted in the Arab countries. The 
evidence gathered from the previous literature suggested that patterns of dementia in the Arab countries are not different from the rest of the countries. However, the prevalence varies from lower sociodemographic status (SES) towards higher SES. The major risk factor that exacerbates dementia among the Arab countries is age and illiteracy. Genetic factors, diabetes mellitus, and CVDs have been identified to also be associated with dementia. Gender differences have not been reported, but a few prevalence studies indicated that females were at a higher risk of dementia, whereas vascular dementia as more common among men. Higher prevalence of dementia and its common risk factors were observed not only among Arabs residing within Arab countries but also among Arab populations living in other countries as well. It is clear from the review that the epidemiology of dementia forms a critical issue that offers a higher scope for research. Future studies should focus on the epidemiology of dementia and other risk factors associated with it, taking into consideration the diversity specific to this region, since the respective differences should be considered in planning healthcare services. The risk factors often vary depending on cultural traditions, social features, demographics, and economic privileges. These differences make the Middle East an important area of research for conducting epidemiological studies to determine the prevalence of certain diseases.

4.1. Implications of Findings for Future Research. A major drawback identified within this study is that dementia is not thought of as a major healthcare challenge [68]. Rather, it is considered a normal aging process [44]. The current demographic transitions of dementia in Egypt have rendered it a future challenge [69]. Similarly, according to the Alzheimer's Disease International (ADI) Delphi consensus study, it was estimated that $71 \%$ of the people living in developing countries will be suffering from dementia by 2040 , which is alarming [70]. The Middle East is characteristically different from other regions in the world in terms of its diversity and heterogeneity in religion. In the Middle East, the elderly population lives among family, with their children and newer generations Therefore, family members should be taught about the symptoms of dementia and how to care for the elderly affected by it. Neuropsychiatric and psychogeriatric specialties can and should play a lead role in this. However, general healthcare should also include the care for $\mathrm{AD}$. The knowledge obtained from this review, and other similar reviews from Arab countries would help public healthcare practitioners and policy makers be able to understand the occurrence and major exacerbating factors related to dementia so as to set priorities and design plans for specialty care for patients with dementia and AD. Longitudinal studies will further aid in understanding the course of diseases, additional symptoms, and problems that can occur with reference to dementia and $\mathrm{AD}$.

\section{Conflicts of Interest}

The authors declare that there are no conflicts of interest regarding the publication of this article.

\section{Acknowledgments}

We would like to thank Miss Laila Mohamed Ghoneim from the American University of Cairo for proofreading and comments that greatly improved the manuscript.

\section{References}

[1] M. Prince, A. Wimo, M. Guerchet, G. Ali, Y. T. Wu, and M. Prina, Alzheimer's Disease International; The global impact of dementia. An analysis of prevalence, incidence, cost and trends, World Alzheimer Report, London, 2015.

[2] J. R. Simpson, "DSM-5 and neurocognitive disorders," Journal of the American Academy of Psychiatry and the Law Online, vol. 42, no. 2, pp. 159-164, 2014.

[3] M. Albugami, N. Qadi, F. Almugbel et al., "The demographic characteristics and the risk factors of dementia in Saudi elderly," American Journal of Psychiatry and Neuroscience, vol. 6, no. 1, article 6531073, pp. 1-8, 2018.

[4] C. Qiu, E. von Strauss, L. Backman, B. Winblad, and L. Fratiglioni, "Twenty-year changes in dementia occurrence suggest decreasing incidence in central Stockholm, Sweden," Neurology, vol. 80, no. 20, pp. 1888-1894, 2013.

[5] World Health Organization and Alzheimer's disease International (ADI), Dementia: a public health priority, World Health Organization, Geneva, 2012.

[6] A. Abyad, "Alzheimer's in the Middle East," Population, vol. 6, p. 12, 2015.

[7] G. H. Suh and A. Shah, "A review of the epidemiological transition in dementia - cross-national comparisons of the indices related to Alzheimer's disease and vascular dementia," Acta Psychiatrica Scandinavica, vol. 104, no. 1, pp. 4-11, 2001.

[8] R. A. Whitmer, E. P. Gunderson, E. Barrett-Connor, C. P. Quesenberry Jr., and K. Yaffe, "Obesity in middle age and future risk of dementia: a 27 year longitudinal population based study," BMJ, vol. 330, no. 7504, p. 1360, 2005.

[9] M. Kivipelto, T. Ngandu, T. Laatikainen, B. Winblad, H. Soininen, and J. Tuomilehto, "Risk score for the prediction of dementia risk in 20 years among middle aged people: a longitudinal, population-based study," The Lancet Neurology, vol. 5, no. 9, pp. 735-741, 2006.

[10] A. Wimo, B. Winblad, H. Aguero-Torres, and E. von Strauss, "The magnitude of dementia occurrence in the world," Alzheimer Disease \& Associated Disorders, vol. 17, no. 2, pp. 63-67, 2003.

[11] F. A. Sosso, O. Nakamura, and M. Nakamura, "Epidemiology of Alzheimer's disease: comparison between Africa and South America," Journal of Neurology and Neuroscience, vol. 08, no. 04, p. 204, 2017.

[12] Economic and Social Affairs of the United Nations Secretariat, World Population Prospects: the 2006 Revisionhttp://www.un .org/esa/population/unpop.htm.

[13] L. Tan, W. Xu, and J. T. Yu, "Meta-analysis of modifiable risk factors for Alzheimer's disease," Journal of Neurology, Neurosurgery \& Psychiatry, vol. 86, 16 Supplement, 2016.

[14] WHO, Background Paper 6.11, Alzheimer disease and other dementias2013, http://www.who.int/medicines/areas/priority_ medicines/BP6_11Alzheimer.pdf.

[15] M. Prince, R. Bryce, E. Albanese, A. Wimo, W. Ribeiro, and C. P. Ferri, "The global prevalence of dementia: A systematic 
review and metaanalysis," The Journal of the Alzheimer's Association, vol. 9, no. 1, pp. 63-75.e2, 2013.

[16] R. Arya, B. Antonisamy, and S. K. Garg, Sample Size Estimation in Prevalence Studies, 2012.

[17] A.-K. F. Farrag, H. M. Farwiz, E. H. Khedr, R. M. Mahfouz, and S. M. Omran, "Prevalence of Alzheimer's disease and other dementing disorders: Assiut-Upper Egypt study," Dementia and Geriatric Cognitive Disorders, vol. 9, no. 6, pp. 323-328, 1998.

[18] H. N. El Tallawy, W. M. Farghaly, T. A. Rageh et al., "Epidemiology of major neurological disorders project in Al Kharga district, New Valley, Egypt," Neuroepidemiology, vol. 35, no. 4, pp. 291-297, 2010.

[19] H. N. El Tallawy, W. M. Farghly, R. Badry et al., "Prevalence of dementia in Al-Quseir city, Red Sea Governorate, Egypt [Corrigendum]," Clinical Interventions in Aging, vol. 9, p. 129, 2014.

[20] E. Khedr, G. Fawi, M. A. Abbas et al., "Prevalence of mild cognitive impairment and dementia among the elderly population of Qena Governorate, Upper Egypt: a community-based study," Journal of Alzheimer's Disease, vol. 45, no. 1, pp. 117126, 2015.

[21] L. M. Chahine, A. Bijlsma, A. P. Hospers, and Z. Chemali, "Dementia and depression among nursing home residents in Lebanon: a pilot study," International Journal of Geriatric Psychiatry, vol. 22, no. 4, pp. 283-285, 2007.

[22] K. T. Phung, M. Chaaya, M. Prince et al., "Dementia prevalence, care arrangement, and access to care in Lebanon: a pilot study," Alzheimer's\&Dementia, vol. 13, no. 12, pp. 13171326, 2017.

[23] S. Ghuloum, A. Bener, and M. T. Abou-Saleh, "Prevalence of mental disorders in adult population attending primary health care setting in Qatari population," Journal of the Pakistan Medical Association, vol. 61, no. 3, p. 216, 2011.

[24] S. Ouanes, F. Fekih-Romdhane, and W. Melki, "Prevalence and management of dementia in nursing home residents in Tunisia," International Journal of Geriatric Psychiatry, vol. 29, no. 8, pp. 877-879, 2014.

[25] R. Ghubash, O. El-Rufaie, T. Zoubeidi, Q. M. Al-Shboul, and S. M. Sabri, "Profile of mental disorders among the elderly United Arab Emirates population: Sociodemographic correlates," International Journal of Geriatric Psychiatry, vol. 19, no. 4, pp. 344-351, 2004.

[26] A. Ogunniyi, A. K. Daif, S. Al-Rajeh et al., "Dementia in Saudi Arabia: experience from a university hospital," Acta Neurologica Scandinavica, vol. 98, no. 2, pp. 116-120, 1998.

[27] N. A. Qureshi, T. A. Al Habeeb, Y. S. Al Ghamdy, M. E. Magzoub, and H. T. Van der Molen, "Psychiatric comorbidity in primary care and hospital referrals, Saudi Arabia," Eastern Mediterranean Health Journal, vol. 7, no. 3, pp. 492501, 2001.

[28] A. L. Hamad and M. A. Ibrahim, "Dementia in Qatar," QNRS Repository, vol. 2011, no. 1, p. 1819, 2011.

[29] M. Chaaya, K. Phung, S. Atweh et al., "Socio-demographic and cardiovascular disease risk factors associated with dementia: results of a cross-sectional study from Lebanon," Preventive Medicine Reports, vol. 9, pp. 1-5, 2018.

[30] E. Al-Khateeb, E. Al-Zayadneh, O. Al-Dalahmah et al., "Relation between copper, lipid profile, and cognition in elderly Jordanians," Journal of Alzheimer's Disease, vol. 41, no. 1, pp. 203-211, 2014.
[31] T. Alaama, M. Basheikh, A. Khiyami, M. Mutwalli, S. Batawi, and G. Watfa, "Diabetes status is associated with poor cognitive performace in Saudi population at high metabolic risk," Journal of Aging Research and Clinical Practice, vol. 5, no. 4, pp. 170-174, 2016.

[32] H. Haithem, A. Ons, N. Salma et al., "Association between dementia and vascular disease-associated polymorphisms in a Tunisian population," International Journal of Neuroscience, vol. 128, no. 1, pp. 32-41, 2017.

[33] C. K. Suemoto, "Dementia prevention and cardiovascular risk factors: a mini review," Principles and Practice of Clinical Research, vol. 1, no. 1, pp. 5-8, 2015.

[34] S. E. Shamieh, C. Costanian, R. Kassir, S. Visvkis-Siest, and N. Bissar-Tadmouri, "APOE genotypes in Lebanon: distribution and association with hypercholesterolemia and Alzheimer's disease," Personalized Medicine, vol. 16, no. 1, pp. 15-23, 2018.

[35] M. Scazufca, P. R. Menezes, H. P. Vallada et al., "High prevalence of dementia among older adults from poor socioeconomic backgrounds in São Paulo, Brazil," International Psychogeriatrics, vol. 20, pp. 394-405, 2008.

[36] A. E. Molero, G. Pino-Ramírez, and G. E. Maestre, "High prevalence of dementia in a Caribbean population," Neuroepidemiology, vol. 29, pp. 107-112, 2007.

[37] J. J. Llibre, M. A. Guerra, H. Pérez-Cruz et al., "Demential syndrome and risk factors in adults over 60 years old living in Havana," Revista de Neurologia, vol. 29, pp. 908-911, 1999.

[38] H. A. De Silva, S. B. Gunatilake, and A. D. Smith, "Prevalence of dementia in a semi-urban population in Sri Lanka: report from a regional survey," International Journal of Geriatric Psychiatry, vol. 18, pp. 711-715, 2003.

[39] M. Je Cho, J. Kyu Kim, and G. H. Suh, "Community study of dementia in the older Korean rural population," Australian \& New Zealand Journal of Psychiatry, vol. 37, pp. 606-612, 2003.

[40] H. Gürvit, M. Emre, S. Tinaz et al., "The prevalence of dementia in an urban Turkish population," American Journal of Alzheimer's Disease \& Other Dementiasr, vol. 23, no. 1, pp. 67-76, 2008.

[41] A. Ertekin, R. Demir, G. Özdemir, L. Özel, E. Özyıldırım, and H. Ulvi, "An investigation of the risk factors and prevalence of Alzheimer's disease in the eastern region of Turkey," European Journal of General Medicine, vol. 12, no. 2, pp. 144-151, 2015.

[42] A. Bowirrat, R. P. Friedland, and A. D. Korczyn, "Vascular dementia among elderly Arabs in Wadi Ara," Journal of the Neurological Sciences, vol. 203-204, pp. 73-76, 2002.

[43] N. Loza and W. Fawzi, "Care for the elderly: the Egyptian experience," Neurobiology of Aging, vol. 35, no. 3, p. 715, 2014.

[44] C. T. Loy, P. R. Schofield, A. M. Turner, and J. B. Kwok, "Genetics of dementia," The Lancet, vol. 383, no. 9919, pp. 828-840, 2014.

[45] A. Ruitenberg, A. Ott, J. C. van Swieten, A. Hofman, and M. M. Breteler, "Incidence of dementia: does gender make a difference?," Neurobiology of Aging, vol. 22, no. 4, pp. 575-580, 2001.

[46] W. A. Rocca, M. M. Mielke, P. Vemuri, and V. M. Miller, "Sex and gender differences in the causes of dementia: a narrative review," Maturitas, vol. 79, no. 2, pp. 196-201, 2014.

[47] P. Vemuri, T. G. Lesnick, S. A. Przybelski et al., "Effect of lifestyle activities on Alzheimer disease biomarkers and cognition," Annals of Neurology, vol. 72, pp. 730-738, 2012. 
[48] A. Bowirrat, R. P. Friedland, L. Farrer, C. Baldwin, and A. Korczyn, "Genetic and environmental risk factors for Alzheimer's disease in Israeli Arabs," Journal of Molecular Neuroscience, vol. 19, no. 1-2, pp. 239-245, 2002.

[49] M. J. Dong, B. Peng, X. T. Lin, J. Zhao, Y. R. Zhou, and R. H. Wang, "The prevalence of dementia in the People's Republic of China: a systematic analysis of 1980-2004 studies," Age and Ageing, vol. 36, no. 6, pp. 619-624, 2007.

[50] O. Alewijn, M. B. Breteler Monique, C. J. J. van HarskampFrans, J. M. van der Cammen Tischa, E. Grobbee Diederick, and A. Hofman, "Prevalence of Alzheimer's disease and vascular dementia: association with education. The Rotterdam study," BMJ, vol. 310, pp. 970-973, 1995.

[51] R. N. Kalaria, G. E. Maestre, R. Arizaga et al., “Alzheimer's disease and vascular dementia in developing countries: prevalence, management, and risk factors. World Federation of Neurology Dementia Research Group," The Lancet Neurology, vol. 7, no. 9, pp. 812-826, 2008.

[52] A. E. Afgin, M. Massarwa, E. Schechtman et al., "High prevalence of mild cognitive impairment and Alzheimer's disease in Arabic villages in northern Israel: impact of gender and education," Journal of Alzheimer's Disease, vol. 29, no. 2, pp. 431439, 2012.

[53] M. H. Eskelinen and M. Kivipelto, "Caffeine as a protective factor in dementia and Alzheimer's disease," Journal of Alzheimer's Disease, vol. 20, no. s1, pp. S167-S174, 2010.

[54] A. Ott, A. J. Slooter, A. Hofman et al., "Smoking and risk of dementia and Alzheimer's disease in a population-based cohort study: the Rotterdam Study," The Lancet, vol. 351, no. 9119, pp. 1840-1843, 1998.

[55] K. P. Muliyala and M. Varghese, "The complex relationship between depression and dementia," Annals of Indian Academy of Neurology, vol. 13, Suppl2, p. S69, 2010.

[56] E. Saedi, M. R. Gheini, F. Faiz, and M. A. Arami, "Diabetes mellitus and cognitive impairments," World Journal of Diabetes, vol. 7, no. 17, p. 412, 2016.

[57] G. J. Biessels, M. W. Strachan, F. L. Visseren, L. J. Kappelle, and R. A. Whitmer, "Dementia and cognitive decline in type 2 diabetes and prediabetic stages: towards targeted interventions," The Lancet Diabetes \& Endocrinology, vol. 2, no. 3, pp. 246255, 2014.

[58] E. Kravitz, J. Schmeidler, and M. S. Beeri, “Type 2 diabetes and cognitive compromise: potential roles of diabetes-related therapies," Endocrinology and Metabolism Clinics of North America, vol. 42, no. 3, pp. 489-501, 2013.

[59] R. Ravona-Springer, X. Luo, J. Schmeidler et al., "Diabetes is associated with increased rate of cognitive decline in questionably demented elderly," Dementia and Geriatric Cognitive Disorders, vol. 29, no. 1, pp. 68-74, 2010.

[60] E. Selvin, S. Bolen, H. C. Yeh et al., "Cardiovascular outcomes in trials of oral diabetes medications: a systematic review," Archives of Internal Medicine, vol. 168, no. 19, pp. 20702080, 2008.

[61] G. Chêne, A. Beiser, R. Au et al., "Gender and incidence of dementia in the Framingham Heart Study from mid-adult life," Alzheimer's \& Dementia, vol. 11, no. 3, pp. 310-320, 2015.

[62] O. Ojo and J. Brooke, "Evaluating the association between diabetes, cognitive decline and dementia," International Journal of Environmental Research and Public Health, vol. 12, no. 7, pp. 8281-8294, 2015.
[63] K. Deckers, M. P. Boxtel, O. J. Schiepers et al., "Target risk factors for dementia prevention: a systematic review and Delphi consensus study on the evidence from observational studies," International Journal of Geriatric Psychiatry, vol. 30, no. 3, pp. 234-246, 2015.

[64] A. Bowirrat, T. A. Treves, R. P. Friedland, and A. D. Korczyn, "Prevalence of Alzheimer's type dementia in an elderly Arab population," European Journal of Neurology, vol. 8, no. 2, pp. 119-123, 2001.

[65] S. D. Israeli-Korn, M. Masarwa, E. Schechtman et al., "Hypertension increases the probability of Alzheimer's disease and of mild cognitive impairment in an Arab community in northern Israel," Neuroepidemiology, vol. 34, no. 2, pp. 99-105, 2010.

[66] M. Badran and I. Laher, "Type II diabetes mellitus in Arabicspeaking countries," International Journal of Endocrinology, vol. 2012, Article ID 902873, 11 pages, 2012.

[67] L. Rizzi, I. Rosset, and M. Roriz-Cruz, "Global epidemiology of dementia: Alzheimer's and vascular types," BioMed Research International, vol. 2014, Article ID 908915, 8 pages, 2014.

[68] D. Arslantas, D. Ozbabalik, and S. Metintas, "Prevalence of dementia and associated risk factors in Middle Anatolia, Turkey," Journal of Clinical Neuroscience, vol. 16, pp. 14551459, 2009.

[69] M. H. Elshahidi, M. A. Elhadidi, A. A. Sharaqi, A. Mostafa, and M. A. Elzhery, "Prevalence of dementia in Egypt: a systematic review," Neuropsychiatric Disease and Treatment, vol. 13, pp. 715-720, 2017.

[70] K. S. Shaji, "Dementia care in developing countries: the road ahead," Indian Journal of Psychiatry, vol. 51, Supplementary 1, p. 5, 2009 . 


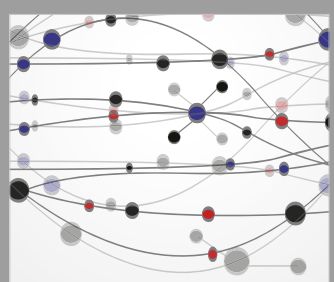

The Scientific World Journal
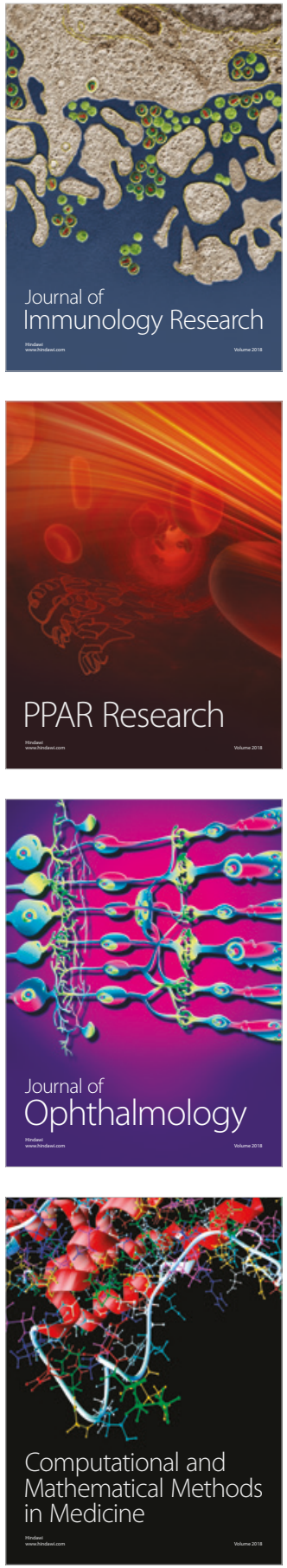

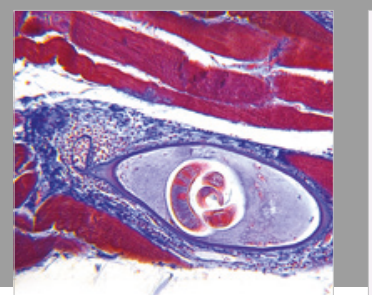

Gastroenterology Research and Practice

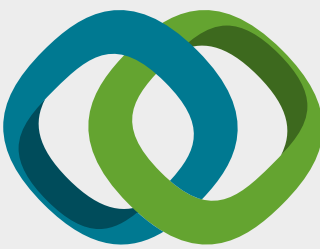

\section{Hindawi}

Submit your manuscripts at

www.hindawi.com
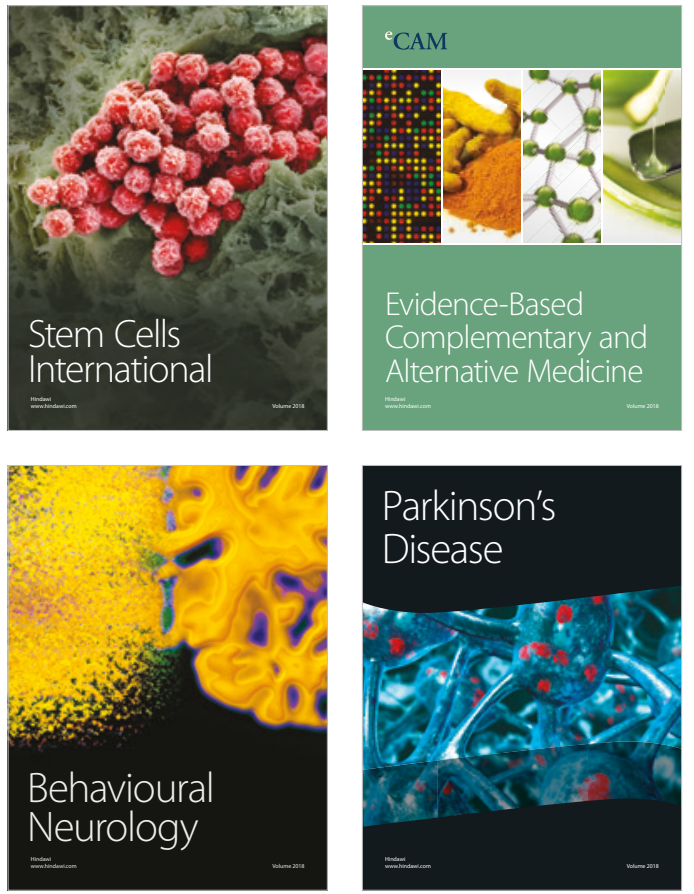

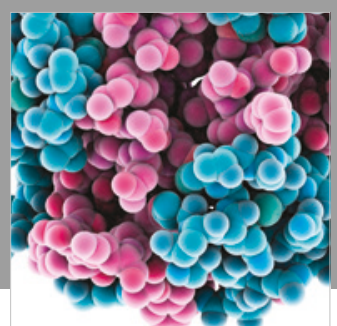

ournal of

Diabetes Research

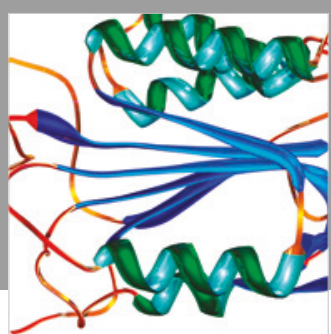

Disease Markers
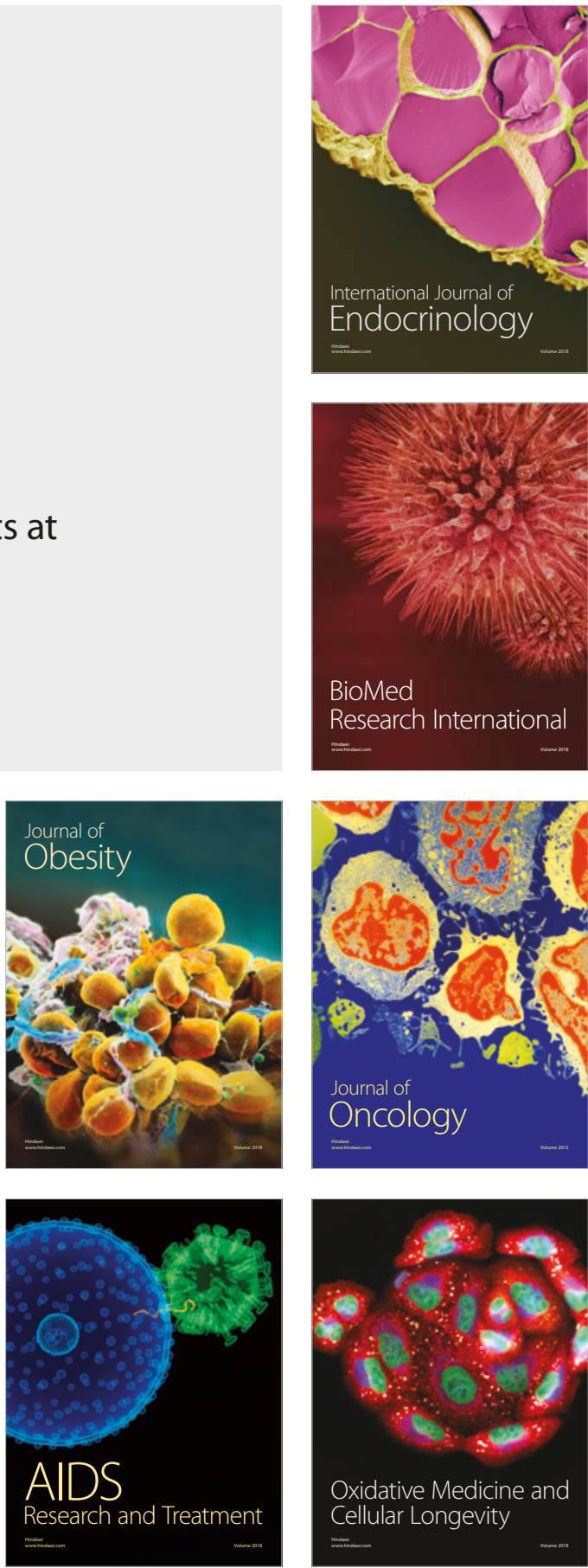